\title{
INFILTRAÇÃO APICAL EM OBTURAÇÕES DE CANAIS REALIZADAS APÓS APLICAÇÃO DO LASER ND:YAG OU SOLUÇÃO DE EDTA NAS PAREDES DO CANAL RADICULAR
}

\author{
APICAL LEAKAGE IN ROOT CANAL FILLINGS PERFORMED WITH TWO \\ TYPES OF CEMENT FOLLOWING APPLICATION OF ND:YAG LASER OR EDTA \\ SOLUTION IN THE ROOT CANAL WALLS
}

\author{
Leandro de Oliveira BASSILI \\ Mestre em Endodontia pela FOB-USP e Coordenador do Curso de Especialização em Endodontia ABO - Niterói. \\ Ivaldo Gomes de MORAES \\ Prof. Dr. Da Disciplina de Endodontia do Departamento de Endodontia, Dentística e Materiais Dentários da FOB-USP.

\section{Raquel Virginia ZANETTI} \\ Profa. Dra. da Disciplina de Prótese da Faculdade da UNICID.
}

Resumo da Dissertação de Mestrado apresentada à Faculdade de Odontologia de Bauru, para obtenção do título de Mestre em Odontologia, área de Endodontia.

\begin{abstract}
A valiou-se a infiltração marginal apical em obturações endodônticas utilizando dois tipos de cimentos após a aplicação do laser Nd:YAG ou solução de EDTA nas paredes do canal radicular. Utilizou-se sessenta e dois dentes pré-molares inferiores humanos que tiveram seus canais radiculares instrumentados por técnica mecanizada. Em seguida, as superfícies externas radiculares foram impermeabilizadas, exceto o forame apical. As raízes foram, então, divididas em 4 grupos de 15, de acordo com o tipo de tratamento realizado nas paredes dos canais radiculares e o tipo de cimento utilizado para a obturação. Grupo I - aplicação do laser Nd:YAG e obturação com cimento AH Plus; Grupo II - aplicação do laser Nd:YAG e obturação com cimento Endofill; Grupo III - aplicação de EDTA por 5 minutos e obturação com cimento AH Plus; Grupo IV - aplicação de EDTA por 5 minutos e obturação com cimento Endofill. Dois dentes serviram como controles positivo e negativo. Em seguida, as raízes foram imersas em solução de azul-de-metileno a $2 \%$, durante 48 horas. Após remoção da impermeabilização foi realizado o desgaste das raízes para leitura da magnitude das infiltrações, por meio de um microscópio óptico pela técnica da planimetria. Os resultados indicaram que o cimento AH Plus foi o que permitiu menor infiltração apical, assim como a aplicação do laser Nd:YAG, quando utilizado no tratamento das paredes dos canais radiculares, anteriormente à obturação.
\end{abstract}

UNITERMOS: Lasers; Ácido Edético; Materiais restauradores do canal radicular.

\section{INTRODUÇÃO}

O tratamento endodôntico tem como principais objetivos, propiciar uma eficiente limpeza do canal radicular a fim de eliminar restos teciduais, microorganismos e ao mesmo tempo dilatá-lo para que seja possível realizar uma obturação tridimensional impermeável, isolando o sistema de canais do resto do organismo.

A efetividade de vários métodos de limpeza dos canais vem sendo estudada, mas a maioria dos pesquisadores concorda que não há um instrumento ou técnica capaz de produzir o alargamento do canal sem que se produza raspas de dentina e, conseqüentemente se forme a smear layer.

A capacidade da smear layer de agir como uma 
barreira física para as bactérias e seus subprodutos, impedindo-os de penetrar na dentina, foi demonstrada por alguns autores ${ }^{6,16}$. Em contra partida, Yamada, et al. ${ }^{22}$, em 1983, e Baker, et al. ${ }^{2}$, em 1975, observaram que as bactérias podem se manter na smear layer e nos túbulos dentinários após a instrumentação dos canais radiculares e, então, sobreviverem e se multiplicarem.

Gettleman, Messer, Eldeeb ${ }^{8}$ (1991); Oksan, et al. ${ }^{17}$ (1993), demonstraram que a smear layer pode agir como uma barreira física, interferindo na adesão e penetração dos cimentos endodônticos para o interior dos túbulos dentinários, e que sua permanência, a longo prazo, comprometeria o selamento apical, pois essa camada se solubilizaria, formando um espaço entre o material obturador e a parede do canal radicular.

Várias são as formas de remoção da smear layer da superfície do canal radicular, sendo o ácido etileno diamino tetraacético (EDTA), a substância mais amplamente utilizada.

Além das substâncias químicas, e do ultra-som, a remoção da smear layer pelo uso dos lasers tem sido demonstrada por alguns autores ${ }^{14,20}$.

Derich, Zakariasen, Tulip ${ }^{5}$, em 1984, foram os primeiros a observarem o derretimento e a fusão da dentina do canal radicular após a aplicação do laser Nd:YAG. Baseados na característica da parede do canal, observada pela microscopia eletrônica de varredura, os autores concluíram que a obliteração dos túbulos dentinários expostos poderia levar a uma diminuição da permeabilidade dentinária.

Park, et al. ${ }^{18}$, em 2001, utilizaram o laser Nd:YAG anteriormente a obturação dos canais radiculares, e concluíram, que os canais obturados dessa forma, mostraram uma menor infiltração apical do que aqueles em que não foi utilizado o laser.

Além do tratamento realizado anteriormente à obturação, o tipo de material obturador a ser utilizado é muito importante. A guta-percha, mundialmente difundida, normalmente, não se adere às paredes do canal radicular, sendo necessária a utilização de um cimento para o preenchimento de todo espaço anatômico do sistema de canais radiculares. $\mathrm{O}$ tipo de cimento utilizado para obturação do canal radicular também irá influenciar no selamento apical, e vários são os trabalhos que mostram a superioridade dos cimentos resinosos para esse fim $^{1,3,12}$.

A literatura é escassa no que diz respeito ao uso do laser Nd:YAG anteriormente à obturação, e os resultados em relação ao selamento apical propiciado por essa técnica.

Este trabalho teve como objetivo avaliar in vitro a influência do tratamento das paredes de canais radiculares, previamente à obturação, com a aplicação do laser de Nd:YAG ou solução de EDTA trissódico, na capacidade seladora de obturações realizadas com dois tipos de cimentos e a possível interação entre os tratamentos e os cimentos utilizados.

\section{MATERIAL E MÉTODOS}

Foram utilizados sessenta e dois pré-molares inferiores humanos extraídos, unirradiculados, com canal único. Os dentes tiveram suas coroas removidas na junção amelo-cementária e, em seguida, procedeuse à padronização do forame apical com lima tipo $\mathrm{K}$ $\mathrm{n}^{\circ} 25$ (Dentsply-Maillefer - Ballaigues, Suiça). Subtraindo-se um milímetro da medida estabelecida, determinou-se o comprimento de trabalho, e os canais foram, então, instrumentados, pela técnica coroa-ápice. Inicialmente, foi introduzida uma lima ProFile 25.06 (Dentsply-Maillefer - Ballaigues, Suiça), acionada pelo motor Endomate (NSK, Japão), no terço cervical e médio do canal, abrindo espaço para utilização das brocas Gates-Glidden 2, 3 e 4, com recuo de $2 \mathrm{~mm}$ entre cada broca utilizada. Posteriormente, progrediuse ao terço apical a partir do instrumento ProFile 25.06, com limas de menores calibres, até atingir o comprimento de trabalho. Nesse comprimento, dilatou-se o terço apical até o instrumento ProFile 35.06 (Dentsply-Maillefer - Ballaigues, Suiça), determinando o diâmetro cirúrgico de instrumentação.

Durante a fase de instrumentação, os canais foram irrigados com uma solução de hipoclorito de sódio a 1\% (Myaco do Brasil Ind e Com. Ltda. SP, Brasil). Ao término dessa, receberam uma irrigação final com solução de soro fisiológico, sendo em seguida secados por aspiração. Na sequiência, as superfícies externas radiculares foram impermeabilizadas com uma camada de Araldite 24 horas e uma camada de esmalte para unhas, respeitando-se as proximidades do forame apical, numa extensão média de $1 \mathrm{~mm}$ ao redor do mesmo.

Após a secagem do agente impermeabilizador, as raízes foram divididas em 4 grupos de 15 , de acordo com o tipo de tratamento ao qual foi submetida a parede do canal radicular e o tipo de cimento utilizado para obturação do sistema de canais radiculares.

Para os grupos I e II foi realizada a aplicação do laser Nd:YAG (SMARTY-A10, Deka, Itália) e as obturações com os cimentos AH Plus (Dentsply De Trey, Konstanz, Alemanha) e Endofill (Dentsply, Petróplis, Brasil) respectivamente. Grupos III e IV foi realizada a aplicação de $10 \mathrm{ml}$ de EDTA trissódico a 17\% (Biodinâmica, Pr, Brasil) por 5 minutos e as 
obturações com os cimentos AH Plus e Endofill respectivamente.

Os parâmetros utilizados para a aplicação do laser Nd:YAG, pulsátil, de $1064 \mathrm{~nm}$ de comprimento de onda, foram de $15 \mathrm{~Hz}$ de frequência, $1.5 \mathrm{~W}$ de potência e $100 \mathrm{~mJ}$ de energia. Para a utilização no interior dos canais radiculares, foi utilizada uma fibra óptica de 200 micrometros de diâmetro. A ponta da fibra era medida e, em seguida, introduzida até o comprimento de trabalho, com o canal seco, e então, o laser era acionado, nos parâmetros anteriormente citados. A seguir, a fibra era removida do interior do canal, em movimento helicoidal, procurando tocar todas as paredes do canal, até atingir o tempo de aplicação. Para cada canal radicular foram feitas duas aplicações de 7,5 segundos, totalizando 15 segundos por canal.

A solução de EDTA trissódico foi aplicada no interior dos canais com auxílio de uma seringa de 5 $\mathrm{ml}$ com agulha hipodérmica, preenchendo-os totalmente e logo após era introduzida uma lima tipo K número 20 (Dentsply-Maillefer - Ballaigues, Suiça) até o comprimento de trabalho, realizando-se uma agitação dessa solução por 1 minuto. Terminado o período de aplicação de 5 minutos, os canais foram irrigados com soro fisiológico e, em seguida, secados para serem obturados.

A obturação dos canais radiculares, para todos os grupos, foi realizada pela associação das técnicas do System B (Analytic Technology, Redmont, WA, USA) e Obtura II (Obtura Corp., Fenton, MO, USA).

Terminada a obturação, a parte mais coronária da raiz foi coberta por um capuz de cera rosa derretida, complementando a impermeabilização.

Para controle da impermeabilização e da própria capacidade de infiltração do corante, duas raízes não tiveram os canais obturados, sendo que uma delas foi totalmente impermeabilizada (controle negativo), inclusive em sua porção apical, enquanto que na outra (controle positivo) apenas o forame apical e $1 \mathrm{~mm}$ ao seu redor ficou livre da impermeabilização.

As raízes foram imersas em solução de azul-demetileno a $2 \%$ durante 48 horas, em estufa a $37^{\circ} \mathrm{C} \pm 2$ e ao final desse período realizou-se a lavagem em água corrente por 12 horas. Em seguida, foi removida a impermeabilização, realizando-se, então, o desgaste da raiz numa das faces proximais até expor a obturação. Para leitura da magnitude das infiltrações, foi utilizado um microscópio óptico (Bausch \& Lomb, EUA) com ocular micrométrica (Carl Zeiss, Alemanha) pela técnica da planimetria. A infiltração marginal apical foi medida, tomando-se como ponto de referência inicial o batente apical até o ponto máximo da infiltração linear do corante, na interface obturação-dentina nas paredes vestibular e lingual. Considerou-se a face de maior infiltração. As medições foram feitas e os dados aferidos foram levados para análise estatística, para aplicação do teste paramétrico análise de variância a dois critérios.

\section{RESULTADOS}

As médias de infiltração marginal apical evidenciada pelo corante azul- de-metileno a $2 \%$ ao longo da interface dentina/obturação, medidas em milímetros, assim como os desvios padrão obtidos em função dos tratamentos da parede do canal radicular e dos cimentos utilizados na obturação estão representados na Tabela 1.

O teste estatístico paramétrico que melhor se adaptou ao modelo experimental foi a análise de variância a dois critérios, representado na Tabela 2.

A análise de variância a dois critérios acusou diferença estatística significante $(p<0,05)$ tanto entre os tratamentos aplicados às paredes do canal radicular previamente à obturação quanto aos cimentos utilizados, apontando valores favoráveis ao laser $\mathrm{Nd}$ :YAG e ao cimento AH Plus, respectivamente, sem, contudo acusar interação entre as variáveis testadas.

\section{DISCUSSÃO}

Ao final da instrumentação, as paredes dos canais radiculares, geralmente, se encontram cobertas pela smear layer, e a necessidade de sua remoção anteriormente à obturação, é controversa ${ }^{2,6,13,22}$.

A eficiência do EDTA na remoção da smear layer da parede dos canais radiculares tem sido demonstrada por alguns autores ${ }^{4,9,19}$. Geralmente, a solução de EDTA trissódico remove a smear layer do terço médio das paredes do canal radicular de uma forma mais eficaz quando comparada ao terço apical ${ }^{23}$.

A utilização do laser na Endodontia vem sendo

TABELA 1- Médias (X) de infiltração marginal apical, em milímetros e, desvio padrão (D.P.), obtidos em função dos tratamentos (Laser, EDTA) e cimentos avaliados (AH Plus, Endofill)

\begin{tabular}{lcccc}
\hline Grupos & Laser - AH Plus & Laser - Endofill & EDTA - AH Plus & EDTA-Endofill \\
X \pm D..P. & $0,99 \pm 0,68$ & $1,93 \pm 1,00$ & $2,54 \pm 0,84$ & $4,20 \pm 1,52$
\end{tabular}


TABELA 2- Análise de variância a dois critérios

\begin{tabular}{lcccccc}
\hline EFEITO & $\begin{array}{c}\text { G.L. } \\
\text { EFEITO }\end{array}$ & $\begin{array}{c}\text { Q.M. } \\
\text { EFEITO }\end{array}$ & $\begin{array}{c}\text { G.L. } \\
\text { ERRO }\end{array}$ & $\begin{array}{c}\text { Q.M. } \\
\text { ERRO }\end{array}$ & F & P \\
\hline TRATAMENTO & $1^{*}$ & $54,645^{*}$ & $56^{*}$ & $1,130^{*}$ & $48,354^{*}$ & $0^{*}$ \\
CIMENTOS & $1^{*}$ & $25,272^{*}$ & $56^{*}$ & $1,130^{*}$ & $22,362^{*}$ & $0^{*}$ \\
INTERAÇÃO & 1 & 1,944 & 56 & 1,130 & 1,720 & 0,195 \\
\hline
\end{tabular}

* - diferença estatisticamente significante $(p<0,05)$

pesquisada há alguns anos, mais precisamente, desde 1971, quando Weichman; Johnson ${ }^{21}$, utilizando o laser $\mathrm{CO}_{2}$, tentaram selar externamente o forame apical de dentes humanos extraídos.

A irradiação do laser Nd:YAG é absorvida pelas estruturas minerais, como os fosfatos e carbonatos da hidroxiapatita, desarranjando a grade cristalina (ablação termoquímica), promovendo o derretimento e a fusão do tecido dentinário. $\mathrm{O}$ tratamento das paredes dos canais radiculares reduz a permeabilidade da dentina, a qual é derretida, selando os túbulos dentinários ${ }^{5,16}$.

Entre os preparos realizados nas paredes dos canais radiculares, previamente à obturação, aquele com o laser Nd:YAG foi o que provocou menor nível de infiltração marginal apical, com diferença estatística significante, em relação ao preparo com EDTA. Esse resultado está de acordo com o trabalho de Goya, et al. ${ }^{10}$, que concluíram que a irradiação das paredes dos canais radiculares com laser $\mathrm{Nd}$ :YAG, principalmente com a associação à tinta nanquim, reduziu de forma significativa a infiltração apical de canais obturados.

Dessa forma, os melhores resultados encontrados em relação à infiltração apical após o uso do laser $\mathrm{Nd}: Y A G$, podem estar relacionados ao fato de uma remoção mais efetiva da smear layer do terço apical do canal por essa técnica, o que permitiria um contato íntimo do material obturador com a parede do canal radicular.

Vários são os trabalhos que mostram a superioridade dos cimentos resinosos em se aderir à dentina ${ }^{7,11}$, e na capacidade de selamento apical ${ }^{3,12}$, quando a smear layer é removida.

Pode-se verificar a superioridade do cimento $\mathrm{AH}$ Plus em comparação ao Endofill no selamento apical, visto que o primeiro apresentou níveis menores de infiltração marginal apical. Esse resultado está de acordo com o trabalho de Almeida et al. ${ }^{1}$, que comparando os cimento Endofill, Ketac-Endo e AH Plus concluíram que esse último obteve menor média de infiltração apical quando comparado com os outros dois, que tiveram resultados estatisticamente semelhantes.

\section{CONCLUSÕES}

Considerando as condições específicas da realização desta pesquisa, com base nos resultados obtidos, podese concluir que:

1) O grupo I (laser + AH Plus), foi o que apresentou menor média de infiltração apical, seguido dos grupos II (laser + Endofill), III (EDTA + Ah Plus) e IV (EDTA + Endofill).

2) $\mathrm{O}$ cimento

AH Plus apresentou melhor capacidade de selamento apical quando comparado com o cimento Endofill ( $\mathrm{p}<$ 0,05), independente do tratamento realizado previamente à obturação.

3) O uso do laser Nd:YAG no interior dos canais radiculares, previamente à obturação, proporcionou menores valores de infiltração apical que a solução de EDTA ( $p<0,05)$, independente do cimento utilizado.

4) Não houve diferenças estatísticas significantes na interação entre os tratamentos da parede dentinária previamente à obturação e os cimentos endodônticos utilizados.

\section{ABSTRACT}

Marginal apical leakage was evaluated in root canal filling performed with two sorts of cements after applying Nd:YAG laser or an EDTA solution in the root canal walls. Sixty-two human lower premolars had their canals instrumented through mechanical technique. Afterwards the external root surfaces were impermeabilized, except for the foramen. The roots were then divided into 4 groups of 15, according to the type of treatment performed on the root canal walls and the type of cement utilized in the filling. Group I application of Nd:YAG laser and filling with AH Plus cement; Group II - application of Nd:YAG laser and filling with Endofill cement; Group III - application of EDTA for 5 minutes and filling with AH Plus cement; Group IV - application of EDTA for 5 minutes and 
filling with Endofill cement. Two teeth served as negative and positive controls. Afterwards, the roots were immersed in a $2 \%$-methylene blue dye, for 48 hours. Wearing of the roots took place following the removal of impermeabilization for the reading of leakage magnitude, by means of a light microscope through planimetry technique The results indicated $\mathrm{AH}$ Plus cement as the one to allow the least apical leakage, as well as Nd:YAG laser application, when utilized in the treatment of root canal walls, prior to filling.

UNITERMS: Lasers; Edetic acid; Root canal filling materials.

\section{REFERÊNCIAS}

1- Almeida WA, Leonardo MR, Tanomaru M, Silva LAB Evaluation of apical sealing of three endodontic sealers. Int Endod J 2000; 33(1):25-7.

2- Baker NA, Eleazer PD, Averbach RE, Seltzer S. Scanning electron microscope study of the efficacy of various irrigant solutions. J Endod 1975; 1(4):127-35.

3- Brandão CG, Moraes IG, Bramante CM. Capacidade seladora apical de cimentos endodônticos ionoméricos. Rev Fac Odontol Bauru 2001; 9:29-34.

4- Ciucchi B, Khettabi M, Holz J. The effectiveness of different endodontic irrigation procedures on the removal of the smear layer: a scanning electron microscopy study. Int Endod J 1989; 22(1):218 .

5- Dederich DN, Zakariasen KL, Tulip J. Scannig electron microscopic analysis of canal wall dentin following neodymiumyttrium-aluminum-garnet laser irradiation. J Endod 1984; 10(9):428-31.

6- Drake DR, Wiemann AH, Rivera EM, Walton RE. Bacterial retention in canal walls in vitro: Effect of smear layer. J Endod 1994; 20(2):78-82.

7- Fidel RAS, Sousa Neto MD, Spano JCE, Barbin EL, Pécora JD. Adhesion of calcium hydroxide-containing root canal sealers. Braz dent J 1994; 5(1):53-7.

8- Gettleman BH, Messer HH, Eldeeb ME. Adhesion of sealer cements to dentin with and without the smear layer. J Endod 1991; 17(1):15-20.

9- Goldberg F, Abramovich A. Analysis of the effect of EDTAC on the dentinal walls of the root canal. J Endod 1977; 3(3):101-5.

10- Goya C, Yamazaki R, Tomita Y, Kimura Y, Matsumoto K. Effects of pulsed Nd:YAG laser irradiation on smear layer at the apical stop and apical leakage after obturation. Int Endod J 2000; 33(3):266-71
11- Grossman LI. Solubility of root canal cements. J Dent Res 1978; 57:927.

12- Haikel Y, Wittenmeyer W, Bateman G, Bentaleb A, Alleman C. A new method for the quantitative analysis of endodontic microleakage. J Endod 1999; 25(3):172-7.

13- Kennedy WA, Walker III WA, Gough RW. Smear layer removal effects on apical leakage. J Endod 1986; 12(1):21-7.

14- Matsuoka E, Kimura Y, Matsumoto K. Studies on the removal of debris near the apical seats by Er:YAG laser and assessment with fiberscope. J Clin Laser Med Surg 1998; 16(5):255-61.

15- Michelich VJ, Schuster JS, Pashley DH. Bacterial penetration of human dentin in vitro. J Dent Res 1980; 59(8):1398-403.

16- Miserendino LJ, Levy GC, Rizoiu IM. Effects of Nd:YAG Laser on the permeability of Root Canal Wall Dentin. J Endod 1995; 21(2):83-7.

17- Oksan T, Aktener BO, Sen BH, Tezel H. The penetration of root canal sealers into dentinal tubules. A scanning electron microscope study. Int Endod J 1993; 26(5):301-5.

18- Park DS, Lee HJ, Yoo HM, Oh TS. Effect of Nd:YAG laser irradiation on the apical leakage of obturated root canals: an electrochemical study. Int Endod J 2001; 34(4):318-21.

19- Ram Z. Chelation in root canal therapy. Oral Surg 1980; 49:6474.

20- Takeda FH, Harashima T, Kimura Y, Matsumoto K. A comparative study of the removal of smear layer by three endodontic irrigants and two types of laser. Int Endod J 1999; 32(1):32-9.

21- Weichman JA, Johnson FM. Laser use in endodontics. A preliminary investigation. Oral Surg 1971; 31(3):416-20.

22- Yamada RS, Armas A, Goldman M, Lin PS. A scanning microscopic comparison of a high volume final flush with several irrigating solutions. J Endod 1983; 9(4):137-42.

23-Zuolo M, Murgel CAF, Pécora JD, AntoniazzI JH, Costa WF. Ação do EDTA e suas associações com tensoativos na permeabilidade da dentina radicular. Rev Odontol USP 1987; $1: 18-23$.

Recebido para publicação em: 25/09/2002

Aceito após reformulações em: 08/04/2003

\author{
Endereço para correspondência: \\ Leandro de Oliveira Bassili \\ Rua Dona Mariana 36, apto 102 \\ Botafogo - RIO DE JANEIRO RJ \\ CEP: 22280-020
}

e-mail: lbassili@terra.com.br 